\title{
Parasitismo florall por abejas sociales (Meliponinae; Apidae) en el árbol quiropterófilo Crescentia alata (Bignoniaceae).
}

\author{
Carlos MARTínez del Río ${ }^{1}$ y STEPhen H. Bullock ${ }^{2}$
}

\begin{abstract}
RESUMEN, Las flores de Crescentia alata son probablemente polinizadas por murciélagos (Glossophaga soricina) y son parasitadas por dos especies de abejas sociales: Partamona bilineata y Trigona fulviventris. Mientras que $P$. bilineata colecta néctar y polen, $T$. fulviventris colecta solamente polen. En cuatro árboles en los que se midió el robo de polen se encontró que la intensidad de éste era mayor en el árbol en el que la dehiscencia de las anteras ocurría más temprano que en aquéllos en los que ocurría más tarde. Las abejas remueven aproximadamente $40 \%$ de la producción tótal de néctar antes de la llegada de los murciélagos. Las abejas actuar como parásitos de las plantas y mantienen una relación amensalistả con los murciélagos. Sugerimos que las plantas polinizadas por murciélagos soh especialmente susceptibles al robo de polen debỉdo a que tienen flores en las que el polen estă expuesto. Además, este polen parece ser especialmente atractivo para las abejas. Las características đe las plantas pólinizadas por murciélagos son probablemente el resultado de la interacción no solamente con polinizadores sino que también con las abejas que parasitan el polen y néctar.
\end{abstract}

ABSTRACT. The flowers of Crescentia alata are probably pollinated by bats (Glossophaga soricina) and parasited by two especies of social bees. Partamona bilineata and Trigona fulviventris. P. bilineata collects pollen and nectar whereas T. fulviventris collects only pollen. In four trees in which pollen robbery was measured it was found that the intensity of robbery was higher in the tree in which anthers dehisced earlier than in those which dehisced later. Bees removed about $40 \%$ of the total production of nectar before the arrival of bats. Bees act as plant parasites and keep an amensalist relationship with bats. We suggest that bat pollinated plants are especially susceptible to pollen robbery because their flowers are open and because the pollen they produce seems to be especially attractive to bees. The traits of bat-pollinated plants may be a result of the interaction not only with pollinators but also with pollen and nectar robbing bees.

1 Department of Zoology, University of Florida, Gainesville Fl, 32611, USA

2 Estación de Chamela, Instituto de Biología, Universidad Nacional Autónoma de México, Apdo. Postal 21, 48980 San Patricio, Jalisco, México

Martínez del Río C, Bullock SH. 1990. Parasitismo floral por abejas sociales (Meliponinae; Apidae) en el árbol quiropterófilo Crescentia alata (Bignoniaceae). Boletín de la Sociedad Botánica de México 50: 69-76. 
La importancia ecológica y evolutiva de los parásitos en asociaciones polinizadorplanta ha sido recientemente reconocida (Soberón y Martínez del Río, 1985). El uso de recompensas florales sin transferencia de polen ha sido coloquialmente llamado 'robo' o 'latrocinio'. El hábito de 'robar' néctar ha recibido considerable atención (Roubik, 1982; McDade y Kinsman, 1980; Inouye, 1980, 1983); el robo de polen, en cambio, ha sido descuidado. La remoción de polen sin transferencia reduce directamente el componente masculino de la fecundidad (Bell, 1985). La remoción de néctar afecta a la adecuación femenina y masculina de las plantas solo indirectamente al alterar los patrones de forrajeo de los polinizadores, y no es posible afirmar a priori si el efecto es positivo o negativo (Soberón y Martínez del Río, 1981).

Las plantas polinizadas por murciélagos son especialmente susceptibles al robo de néctar y polen debido a que tienen flores abiertas en las que el polen y néctar están relativamente expuestos. La presencia de abejas colectoras de polen en plantas quiropterófilas ha sido reportada en numerosas ocasiones (Baker et al., 1971; Lack, 1978; Eguiarte et al., 1987). Eguiarte et al. (1987) informaron la presencia de numerosas abejas en las flores recién abiertas de Pseudobombax ellipticum (Bombacaceae) y sugirieron que la existencia de robadores de polen era una de las influencias determinantes en la hora de antésis en plantas quiropterófilas. Numerosas aves visitan a las flores quiropterófilas durante el día en busca del néctar no utilizado por los murciélagos. Eguiarte y Búrquez (1987) han sugerido que la remoción de polen que las abejas efectúan reduce el efecto potencial de estos polinizadores diurnos en las plantas. El efecto de las abejas sociales puede ser severo para las plantas debido a que el polen es usado por las abejas como alimento para las larvas, y por lo tanto representa un recurso sumamente importante (Michener, 1974). Las abejas en la tribu Meliponini, poseen la capacidad de reclutar nuevos individuos muy rápidamente (Hubbell y Johnson, 1978) y forman colonias que pueden incluir miles de individuos (Michener, 1974).

En contraste con las Agavaceae y Bombacaceae polinizadas por murciélagos que tienen flores abiertas y que producen abundante polen (Eguiarte y Búrquez, 1987; Eguiarte et al., 1987; Martínez del Río y Eguiarte, 1987), las Bignoniaceae quiropterófilas poseen flores tubulares y producen relativamente poco polen. Aquí presentamos algunos datos cuantitativos sobre el robo de polen y néctar por dos especies de meliponinos (Partamona bilineata(Say) y Trigona fulviventris Guerín) a las flores de una Bignoniaceae polinizada por murciélagos: Crescentia alata HBK. Nuestros datos sugieren que en esta planta las abejas reducen la disponibilidad de néctar para los murciélagos y que el efecto sobre la adecuación masculina de las plantas puede ser muy severo.

\section{MÉTODOS}

Las observaciones se realizaron durante el mes de julio en la Estación de Biología "Chamela", Instituto de Biología, UNAM, en el estado de Jalisco, México. El clima es 
monzónico (Bullock, 1986) y la selva es excepcional por su riqueza de especies leñosas (Lott et al., 1987). C. alata es un árbol común en las selvas subcaducifolias que bordean los lechos secos de los arroyos y las cañadas, pero también se le puede encontrar en la vegetación caducifolias que cubre las serranías.

Con el objeto de determinar la producción de néctar, 5-9 flores a punto de abrir fueron cubiertas con bolsas de papel en cada uno de 4 árboles separados por 1-3km. Comenzando a las 17:30 hrs. el volumen de néctar producido por estas flores fue extraído y medido a intervalos irregulares con jeringas desechables. El néctar era cuidadosamente redepositado en la flor después de cada medida y la flor volvía a ser cubierta. El efecto de los robadores sobre la disponibilidad de néctar fue estimado midiendo a las 20:00 hrs. el volumen contenido en muestras aleatorias de 10 flores no cubiertas en cada uno de los árboles. La concentración de néctar fue medida en 30 flores no cubiertas usando un refractómetro de campo.

Para estimar el robo de polen se escogieron 20 flores al azar en 4 árboles y cada 10 min., comenzando a las 17:00 hrs., se contó el númerơ de tecas abiertas (dehiscentes), tecas dañadas (dado que con frecuencia laš abejas mordían las anteras antes de que éstas abrieran), y tecas vacías. Tecas vacías son aq̨uellas que no contenían cantidades visibles de polen. Dado que los murciélagos qo comenzaban a visitar a las flores sino hasta aproximadamente las 20:15 hrs. asumimos que la remoción de polen ocurrida antes de las 20:00 hrs. era debida exclusivamente a la actividad de las abejas. Todas las especies de abejas observadas visitando las flores fueron colectadas e identificadas. Usando redes colocadas en las cercanías de árbolę́s' en floración capturamos murciélagos para posterior identificación.

\section{RESULTADOS}

Las flores de C. alata son tubulares y caulifloras con cuatro anteras insertas. Durante el mes de julio en Chamela abren entre las 18:30 y las 19:00 hrs. Después el perianto se desprende y cae entre las 05:00 y las 06:00 hrs. del día siguiente. Desde el momento en que las flores abren hasta el crepúsculo, que ocurre a las 20:00 hrs. las flores son visitadas por dos especies de abejas sociales Trigona fuliviventris y Partamona bilineata. En cuanto las flores abren, ambas especies dañan las anteras usando las mandíbulas. Las abejas remueven polen de las tecas dañadas en pequeñas cantidades. En cuanto las anteras abren, las abejas remueven el polen rápidamente. Ambas especies de abejas colectan polen pero solamente $P$. orizabaensis roba néctar. Los murciélagos comienzan a visitar las flores poco después del crepúsculo, la hora promedio de la primera visita en 7 noches es las 20:11 hrs. (rango = 20:07-20:21). Durante dos noches en que se colocaron redes abajo de árboles en floración capturamos exclusivamente a Glossophaga soricina (Glossophaginae, Phyllostomidae; 15 individuos en 12 hrs./red, 6 hrs. por noche). En 20 hrs. 
de observación (entre las 17:30 y las 20:00 hrs.) solamente en dos ocasiones observamos colibríes (Amazilia rutila, Trochilidae) visitando a las flores.

Como la mayoría de las especies polinizadas por murciélagos las flores de C. alata produen una gran cantidad de néctar diluído (concentración promedio $\pm \mathrm{DE}=13,9 \pm$ $1.2 \%$ peso/peso total, $\mathrm{N}=50$ ). El volumen promedio total acumulado por noche por flor es 480 microlitros (error estándar de la media $(\mathrm{EE})=13.5, \mathrm{~N}=35$, media ponderada de 4 árboles). El patrón cóncavo de acumulación de néctar sugiere que la tasa de producción de néctar es máxima durante las primeras horas de la noche y decrece de ahí en adelante (figura 1). La cantidad promedio de néctar presente en flores no cubiertas a las 20:00 hrs. es $1.4 \mu \mathrm{l}(\mathrm{EE}=0.41, \mathrm{~N}=40,10$ flóres por árbol en 4 árboles $)$. En contraste, la cantidad promedio de néctar en flores cubiertas es $196 \mu 1$ ( $\mathrm{EE}=4.54$, $\mathrm{n}=38$, media ponderada de 4 árboles). Las abejas, en consecuencia, remueven aproximadamente el $40 \%$ del néctar total producido por flor.

Entre el $83 \%$ y el 100\% de las tecas examinadas a las 19:00 hrs. mostraron signos de daño por abejas $(\mathrm{N}=4$ árboles, 20 flores y 80 tecas por árbol). El porcentaje acumulativo de tecas abiertas y de tecas vaciadas por las abejas a lo largo de la tarde se muestra en las figuras $2 \mathrm{a}$ y $2 \mathrm{~b}$. En el árbol 1 en el que las tecas abren más temprano el $84 \%$ de las tecas se encuentran vacías a las 20:00 hrs. En el árbol 2 las valvas abren más tarde y solamente el 13\% de las anteras se encontraban vacías a las 20:00 hrs. Los árboles 3 y 4 son intermedios entre los árboles 1 y 2 y muestran porcentajes intermedios de robo ( $40 \%$ y $52 \%$ respectivamente). El patrón sugiere que aquellos árboles que abren mâs temprano sufren un mayor robo de polen por parte de las abejas.

\section{DISCUSIÓN}

Los datos presentados sugieren que los robadores pueden reducir la disponibilidad de polen y néctar considerablemente. Al reducir la cantidad de polen y néctar disponible para los murciélagos, las abejas mantienen con éstos una relación neegativa y asimétrica que puede ser clasificada como amensalismo. El efecto de los murciélagos sobre la disponibilidad de polen y néctar para las abejas es nulo debido a que tos murciélagọs comienzan a visitar las flores después del crepúsculo y, a que la corola de las flores caẹ antes del amanecer.

La reducción en la cantidad de polen que resulta de la actividad deilas abejas afecta de un modo directo al componente masculino en la fecundidad de las plantias. El efecto del robo de polen puede ser muy severo como lo ejemplifica el árbod 1 en el que más del $80 \%$ de polen fue removido antes de la aparición del primer polinizador. Al reducir lá cantidad de néctar por flor las abejas posiblemente modifican los patrones de forrajeo de los murciélagos e indirectamente la polinización (por ejemplo, Roubik et al; 1985). Es difícil, sin embargo predecir si el efecto sobre las plant as es positivo (aumento en el flujo de polen entre plantas) o negativo (reducción en el flujo de polen, ver Soberón y 


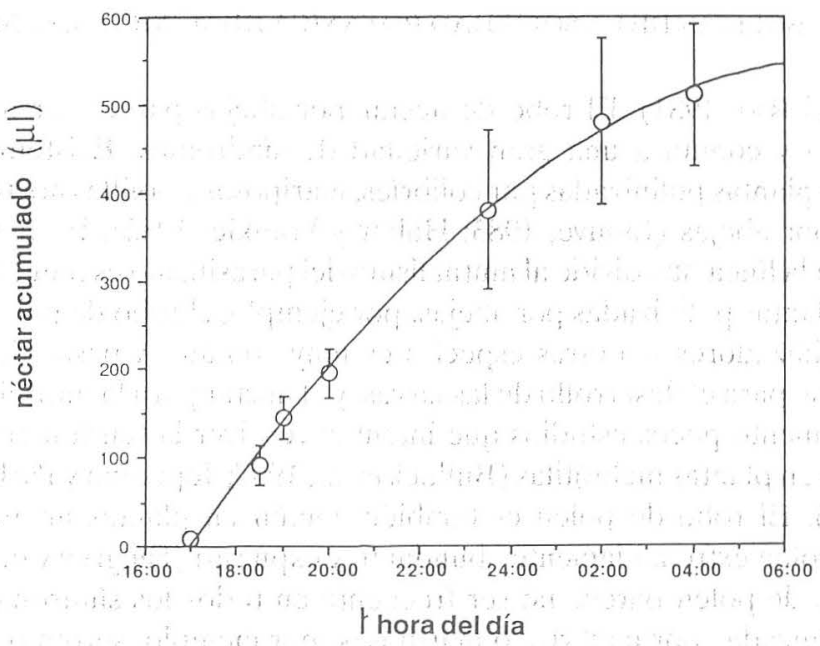

Fig. 1. Curva de acumulación de néctar. Cada punto es la medida ponderada de 4 árboles (5-9 flores por árbol) las barrạs son errores estándar de la media. La curva mostrada es un polinomio de segundo grado ajustado a los datos por mínimos cuadrados y presentado exclusivamente con fines descriptivos.
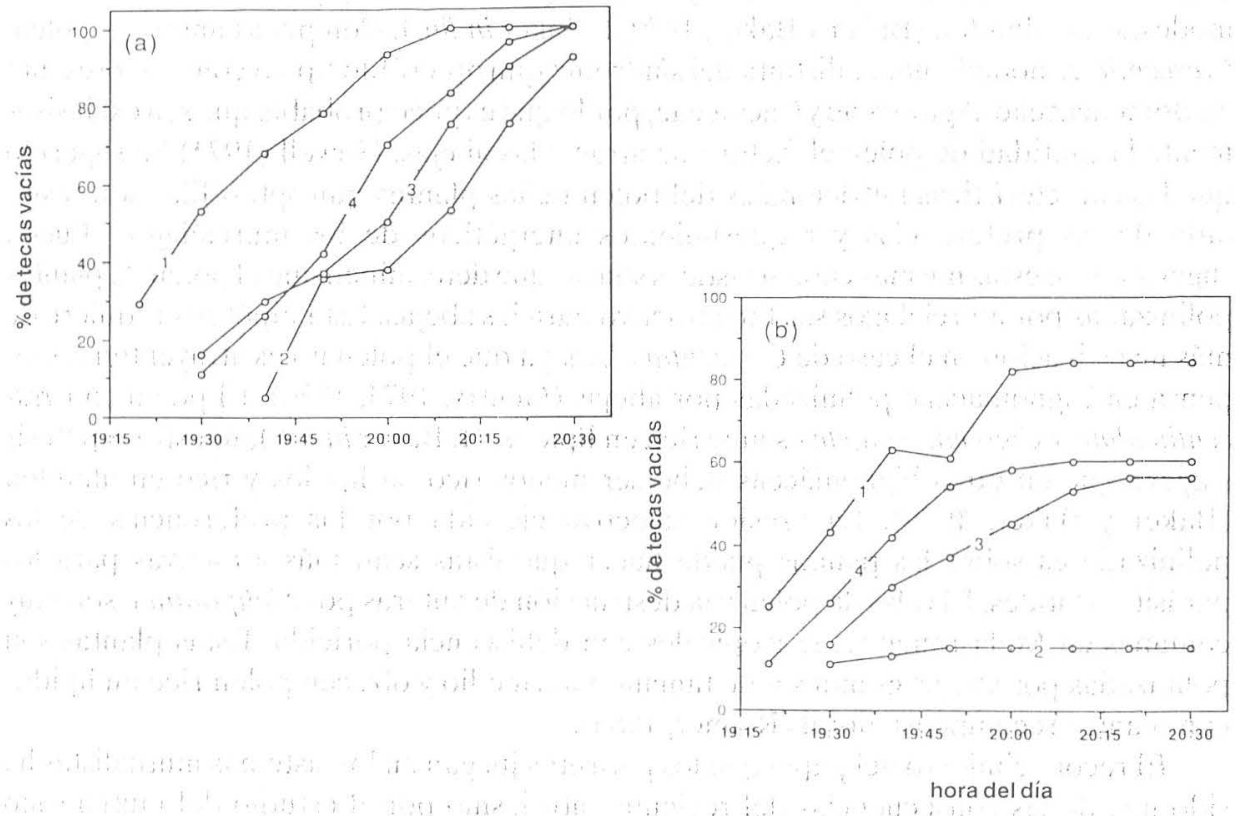

Fïg. 2. Dehiscencia de anteras y robo de polen: a) distribución acumulađa de apertura de anteras cono función del tiempo en 4 árboles de C. alata, b) distribución acumulada de tecas vacías como función del tiempo. Cada curva está basada en una muestra de 80 tecas (20 flores). Cada curva representa un árbol. 
Martínez del Río, 1981). El robo de néctar por abejas parece ser un fenómeno muy generalizado y común a una gran variedad de síndromes. Existen una multitud de ejemplos de plantas polinizadas por colibríes, mariposas y polillas nocturnas cuyo néctar es robado por abejas (Inouye, 1983; Haber y Frankie, 1982). El algunos sistemas de polinización la línea que divide al mutualismo del parasitismo es ténue (Howe y Westley, 1988). En plantas polinizadas por abejas, por ejemplo, el robo de polen y néctar por los mismos polinizadores y/o otras especies es muy común. A pesar de que el polen es indispensable para el desarrollo de las larvas, y el néctar para la nutrición de los adultos, hay relativamente pocos estudios que intentan resolver la tensión entre parasitismo y mutualismo en plantas melitófilas (Bullock et al., 1987; Tepedino y Parker, 1982; Bullock et al., 1989). El robo de polen es también común en plantas anemófilas, las cuales presentan polen extremadamente abundante y expuesto (Faegri y van der Pijl, 1979).

El robo de polen parece no ser frecuente en todos los síndromes florales. Pocas plantas polinizadas por colibríes o mariposas, por ejemplo, sufren de robo intenso de polen por abejas (Martíneż del Río obs.pers). Las plantas polinizadas por murciélagos pueden ser utilizadas como fuentes de polen muy apetecidas por abejas porque el polen se produce en grandes cantidades, está expuesto, y/o porque contiene concentraciones relativamente altas de aminoácidos y lípidos (Howell, 1974) y contiene cantidades modestas de almidón (Baker y Baker, 1979; I. Baker in litt.). Con pocas anteras y polen, Crescentia es notablemente distinta del síndrome quiropterófilo típico como se presente en Bombacaceae, Agavaceae y Cactaceae, por lo que es poco probable que sea exclusivamente la cantidad de polen el factor que atrae a las abejas. Howell (1974) ha sugerido que las características nutricionales del polen de las plantas quiropterófilas son resultado de las preferencias y requerimientos energéticos de los murciélagos. Puede sugerirse que estas mismas características son las que determinan que el polen de plantas polinizadas por murciélagos sea tan atractivo para las abejas. Esta hipótesis requiere de más investigación en el caso de Crescentia alata, ya que el polen no es atrayente/recompensa en Bignoniaceae polinizadas por abejas (Gentry, 1974, 1976). El polen en Crescentia alata y Crescentia cujete es muy rico en lípidos. (I. Baker in litt.); nuestra hipótesis sugiere que en otras bignoniáceas debe ser menos rico en lípidos y rico en almidón (Baker y Baker, 1979). La presión selectiva ejercida por las preferencias de los polinizadores sobre las plantas puede hacer que éstas sean más atractivas para los parásitos florales. El robo de polen y la destrucción de anteras por Meliponinae son muy comunes en Melastomataceae tropicales con dehiscencia poricida. Estas plantas son polinizadas por abejas grandes o de tamaño intermedio y ofrecen polen rico en lípidos como única recompensa floral (Renner, 1983).

El reconocimiento del papel que los parásitos juegan en los sistemas mutualistas ha sido una de las consecuencias del reciente entusiasmo por el estudio del mutualismo (Soberón y Martínez del Río, 1985). Nuestros resultados enfatizan la importancia que los parásitos florales y especialmente los robadores de polen tienen en la asociación polinizador-planta y en particular sugieren que el parasitismo floral por abejas puede 
jugar un papel determinante en la evolución de las características de las plantas polinizadas por murciélagos.

AGRADECIMIENTOS. Ricardo Ayala ayudó a identificar a las abejas y Héctor Arita a los murciélagos. Carolina Murcia, Héctor Arita y dos revisores anónimos criticaron una versión preliminar del manuscrito. Los datos contenidos en este trabajo fueron reunidos en gran parte gracias a las ideas y al estímulo de Luis Eguiarte.

\section{LITERATURA CITADA}

BELL, G. 1985. On the function of flowers. Proc. Roy. Soc. London, Ser. B, Biol, Sci. 224:223-265.

BAKER, H. G. y I. BAKER. 1979. Starch in angiosperm pollen grains and its evolutionary significance Amer. J. Bot. 66:591-600.

BAKER, H. G., R. W. CRUDEN y I. BAKER. 1971. Minor parasitism in pollination biology and its community funtion: the case of Ceiba acuminata. Bioscience 21:1127-1129.

BULLOCK, S. H. 1986. Climate of Chamela, Jalisco and trends in the south coastal region of Mexico. Arch. Met. Geop. Biocl. Ser. B 36:297-316.

BULLOCK, S. H., R. AYALA, I. BAKER y H. G. BAKER. 1987. Reproductive biology of Ipomoea wolcottiana (Convolvulaceae). Madroño 34:304-314.

BULLOCK, S. H., C. MARTínEZ DEL RÍO y R. AYALA. 1989. Bee visitation rates to trees of Prockia crucis differing in flower number. Oecologia 78:389-393.

EGUIARTE, L. E. y A. BÚRQUEZ. 1987. Reproductive ecology of Manfreda brachystackia, an iteroparous species of Agavaceae. Southw. Naturalist 32:169-178.

EGUIARTE, L. E., C. MARTÍNEZ DEL RÍO y H. ARITA. 1987. El polen y néctar como recursos: el papel ecológico de los visitantes a las flores de Pseudobombax ellipticum. Biotropica 19:74-82.

FAEGRI, K. y L. VAN DER PIJL. 1979. Principles of pollination ecology. 3a. ed. Pergamon Press, Oxford.

GENTRY, A. H. 1974. Co-evolutionary patterns in Central American Bignoniaceae. Ann. Missouri Bot. Gardl. 61:728-759.

GENTRY, A. H. 1976. Bignoniaceae of southern Central America: distribution and ecological specificity. Biotropica 8:117-131.

HABER, W.A. y G. W. FRANKIE. 1982. Pollination of Luehea (Tiliaceae) in Costa Rican deciduous forest. Ecology 63:1740-1751.

HOWE, H. F. y L. C. WESTLEY. 1988. Ecological relationships of plants and animals. Oxford University Press.

HOWELL, D. J. 1974. Bats and pollen: physiological aspects of the syndrome of chiropterophily. Comp. Biochem. Physiol. 48A:263-276.

HUBBELL, S. P. y L. K. JOHNSON. 1978. Comparative foraging of six stingless bee species exploiting a standardized resource. Ecology 59:1123-1136.

INOUYE, D. W. 1980. The terminology of floral larceny. Ecology 61:1251-1253.

INOUYE, D. W. 1983. The ecology of nectar robbing. En: Bentley, B. y T. Elias (Edrs.). The Biology of nectaries. Columbia University Press, New York. pp. 153-173.

LACK, A. 1978. The ecology of the flowers of the savanna tree Maranthes polyandra and their visitors, with particular reference to bats. J. Ecol. 66:287-295.

LOTT, E. J., S. H. BULLOCK y J. A. SOLIS. Floristic diversity and structure of upland and arroyo forests in coastal Jalisco. Biotropica 19:228-235. 
MARTÍNEZ DEL RÍO, C. y I. E. EGUIARTE. 1987. Bird visitation to Agave salmiana: comparison among hummingbirds and perching birds. Condor 89:357-363.

McDADE, L. A. y S. KINSMAN. 1980. The impact of floral parasitism in two neotropical humminingbird pollinated plant species. Evolution 34:944-958.

MICHENER, C. D. 1974. The social behavior of the bees: a comparative study. Harvard University Press, Boston.

RENNER, S. 1983. The widespread occurrence of anther destruction by Trigona bees in Melastomataceae. Biotropica 15:257-267.

ROUBIK, D. W. 1982. The ecological impact of nectar-robbing bees and pollinating hummingbirds on a tropical shrub. Ecology 63:354-360.

ROUBIK, D. W., N. M. HOLBROOK y G. PARRA. 1985. Roles of nectar robbers in reproduction of the tropical treelet Quassia amara (Simaroubaceac). Oecologia 66:161-167.

SOBERÓN, J y C. MARTÍNEZ DEL RÍO. 1981. The dynamics of a plant-pollinator interaction. J. Theor. BBiol. 91:363-378.

SOBERÓN, J y C. MARTÍNEZ DEL RÍO. 1985. Cheating and taking advantage in mutualistic systems. En: Boucher, D. II. (Edr.) The biology of mutualism. Croom-Hclm, London. pp. 192-216.

TEPEDINO, V.J. y F. D. PARKER 1982. Interspecific differences in the relative importance of pollen and nectar to bee species foraging on sunflowers. Environ. Entomol. 11:246-250. 